\title{
Entrelacs
}

Cinéma et audiovisuel

13 | 2017

Espace, perspective et fragmentation

\section{Espace, Perspective et Fragmentation Se jouer de l'œil ou pas. Entre modèle et imaginaire}

\section{Grégory Bled}

\section{(2) OpenEdition}

\section{Journals}

Édition électronique

URL : http://journals.openedition.org/entrelacs/2049

DOI : 10.4000/entrelacs.2049

ISSN : 2261-5482

Éditeur

Éditions Téraèdre

Référence électronique

Grégory Bled, « Espace, Perspective et Fragmentation Se jouer de l'œil ou pas. Entre modèle et imaginaire », Entrelacs [En ligne], 13 | 2017, mis en ligne le 05 mars 2017, consulté le 24 septembre 2020. URL : http://journals.openedition.org/entrelacs/2049; DOI : https://doi.org/10.4000/entrelacs 2049

Ce document a été généré automatiquement le 24 septembre 2020.

Tous droits réservés 


\title{
Espace, Perspective et Fragmentation Se jouer de l'œil ou pas. Entre modèle et imaginaire
}

\author{
Grégory Bled
}

1 À la lecture des textes présentés dans cet ouvrage, il ressort évidemment une matrice de fond, une structure, un mot: l'œil, c'est par lui que nous sommes conviés au spectacle de l'espace et à la lecture des images. Fin $\mathrm{X}^{\mathrm{e}}$-début $\mathrm{XI}^{\mathrm{e}}$ siècle, les travaux du savant arabe Ibn al-Haytham, connu en occident sous le nom d'Alhazen mettent en évidence une notion de sens à distance ${ }^{1}$, la vue n'est plus toucher, palpation, mais réception ; les rayons visuels sortant de l'œil ne viennent plus palper l'objet, mais les rayons lumineux y pénètrent et c'est dans le corps, sur la surface du cristallin que la sensation du monde extérieur vient se fixer. Pour Gérard Simon «La lumière est nécessaire à la vision $»^{2}$ il $\mathrm{y}$ a donc nécessité à la mettre en scène. Cette théorie de l'intromission est une naissance de dispositifs scénographiques (lumière, espace) de la peinture à la scène théâtrale et au cinéma.

2 Les artistes, plasticiens, scénographes, architectes connaissent l'intérêt de cet œil récepteur (œil-artiste et celui du spectateur-relais), ils ont cette lourde responsabilité de montrer aux spectateurs, aux utilisateurs leurs visions de la chose. Certains montrent, reproduisent ce qu'ils voient, se plient aux lois de la perspective, et en cela ils rejoignent ces "vidéastes" (je vois, j'enregistre, et je restitue) de la Renaissance. D'autres rejettent cette représentation ${ }^{3}$ de l'espace évidente et simple, redécouvrent ou inventent d'autres codes, «C'est au moment où le type de langage figuratif qui s'est élaboré au moment de la Renaissance cesse de satisfaire à tous les besoins de la pensée et de la sensibilité moderne qu'il est permis, me semble-t-il, de se retourner vers les origines $»^{4}$.

3 Ils retournent les yeux vers l'intérieur, en direction de leurs mémoires, de leurs rêves, se libèrent de cet œil copieur ${ }^{5}$ qui pour Hegel ne sert qu'à l'imitation, et n'aura jamais la valeur de la création. Libérés de l'œil, ils ont un regard symbolique, en cela ils peuvent s'apparenter aux artistes du Moyen Âge ${ }^{6}$ qui reproduisaient l'empreinte de l'objet 
laissée dans leurs mémoires plutôt que l'objet vu. Ces artistes savent concrétiser cette vision que Lucrèce nomme vision de l'esprit. Pour Lucrèce, cette vision de l'esprit est comparable à celle des yeux "sauf qu'il (l'esprit) distingue des images plus subtiles encore", «c'est ainsi que nous voyons des Centaures, des formes de Scyllas, des museaux de Cerbères, et les fantômes des morts dont la terre recouvre les os $»^{7}$, Lucrèce précise plus loin que l'image du Centaure ne provient pas d'un modèle puisqu'un tel animal n'a jamais existé.

4 Les Arts ont su nous décrire, illustrer, un grand nombre de monstres, de créatures fantastiques, d'espaces extraordinaires. Le cinéma dès ses débuts a compris le potentiel illustratif qu'il possède, Méliès nous fera visiter les fonds marins peuplés d'étranges créatures, il nous conviera à un voyage dans la lune, dans un espace de carton-pâte, de toiles peintes. Alain Resnais avait l'habitude de dire que le cinéma c'était les frères Lumières et Méliès, lui se sentait proche de Mélièss, il rajoutait aussi: "Je reste sûrement, par mes goûts, un metteur en scène d'avant-guerre, j'en suis conscient et je n'ai jamais eu peur du studio. C'est impressionnant un studio, on peut tout y faire $»^{9}$. Voilà tout faire, tout se permettre et donc choisir entre le tout-œil ou le non-œil, le modèle vu, repéré en un nombre infini de coordonnées spatiales ${ }^{10}$ ou l'empreinte du modèle laissée par des conteurs merveilleux comme en parle Roland Barthes dans un texte sur Arcimboldo ${ }^{11}$.

\section{La représentation d'un espace qui se joue de l'œil}

Quel point commun peut-il y avoir entre la metteuse en scène Tatiana Stépantchenko, les artistes Fernand Léger et Christian Boltanski ? La première, dans sa représentation plastique du château d'Elseneur de Hamlet ${ }^{12}$, pense l'espace libéré de ses murs et de son opacité. Elle impose une transparence, un rythme de lignes de forces, de structures, invente une perspective. Elle nous confronte au lieu du château qui devient un « lieu de réclusion faite de lamelles élastiques dont la verticalité et les couleurs renvoyaient à des barreaux de prison $»^{13}$. Fernand Léger lui «lors de la réalisation du film Ballet mécanique, utilisait et mettait en scène des artifices, tels que des miroirs et des objets réfléchissants, en vue d'introduire des "structures de vertige " et de provoquer des "affolements visuels ${ }^{14}$. L'artiste Christian Boltanski dans son théâtre d'ombres nous replonge dans ces espaces délicieux de nos chambres d'enfant où l'ombre projetée ${ }^{15}$, déformée, anamorphosée, matérialise en quelque sorte le lieu, le lieu du rêve, espace indécis.

6 Ces trois créateurs sont des amoureux de l'espace et de sa représentation, ils aiment le fragmenter et le déstructurer, la scène devient « scène d'invention totale $»^{16}$. Ils ont su voir autrement et nous montrer autrement, ils ont ce regard symbolique. Ils se sont libérés de la dictature d'une vision contenue, orientée. Cette perspective symbolique où l'intérêt et le signe sont plus importants qu'une logique visuelle sujette à la profondeur offre à l'artiste de nombreuses possibilités de représentation spatiale. Ces artistesplasticiens maitrisant ces lois de la "perspective du spectacle " s'amusent, jouent de ses propriétés, la boite noire y devient une boite d'espaces ${ }^{17}$. La scène théâtrale se couvre de projections cinématographiques, en un mélange créant un lieu narratif, tels les dispositifs scénographiques de la compagnie chilienne Teatrocinema, qui comme son nom l'indique, « cherche littéralement à fondre les langages et les techniques des deux arts, pour qu'ils deviennent inséparables et uniques $»^{18}$. 
7 L'expression de toute cette sensibilité spatiale peut se passer du tout-œil, il faut plus que l'œil pour peindre, concevoir un espace, se jouer des grandeurs et du rapport entre les figures. Il faut travailler avec cet outil de captation avec parcimonie pour très vite l'oublier. La première raison pour oublier cet outil est bien de le considérer comme un outil commun, l'artiste fabrique ses outils, taille sa brosse, affine un roseau, il doit donc modifier son œil et voir autrement pour apprendre au spectateur à voir bien plus.

8 Il faut se libérer et la difficulté est là : échapper à ce qu'Augustin Berque nomme le 《 voir comme $»^{19}$, cette vision particulière, « une subjectivité ; c'est-à-dire à la manière dont une certaine société voit le monde, et ce faisant l'interprète et le modèle en fonction de ce voir-comme ». Augustin Berque cite Cézanne qui, parlant de la montagne Sainte Victoire, écrivait que les paysans de la région d'Aix ne voyaient pas la montagne comme un paysage. "Il faut un certain goût, un certain voir-comme, lequel ne s'acquiert que par une certaine culture $»^{20}$.

9 Pour ces artistes, à l'image de Cézanne, la figure peut être déformée, représentée de façon fragmentaire, elle doit juste signifier, le trait laisse sa place à la couleur, l'espace ne sera plus construit à l'aide de tracés savants, c'est la juxtaposition de schèmes de couleur qui créera un espace, une forme. Émile Bernard nous dira sur Cézanne :

Lire la nature, c'est la voir sous le voile de l'interprétation par taches colorées se succédant selon une loi d'harmonie. Ces grandes teintes s'analysent ainsi par les modulations. Peindre c'est enregistrer ses sensations colorées. Il n'y a pas de ligne, il n'y a pas de modelé, il n'y a que des contrastes. Ces contrastes, ce ne sont pas le noir et le blanc qui les donnent, c'est la sensation colorée. ${ }^{21}$

10 Tout voir derrière le voile de l'interprétation, filtrer son regard, puis laisser notre imagination dans une ambulatio pénétrer de nouveaux espaces, de nouvelles perspectives, créer de nouvelles tensions, de nouvelles émotions. Cette création d'émotions ne laissera pas indifférent, elle sera un substrat à une gestion sensible de l'espace, c'est-à-dire à ce moment particulier où face à un espace, il est donc constructif de cligner des yeux afin d'en avoir une lecture simplifiée que je pourrais appeler abstraite, en faire un assemblage de masses, de zones, d'aplats de couleurs, sentir si cela fonctionne. L'équilibre abstrait est peut-être ce par quoi l'ordonnancement du jardin nous invite ou pas à la promenade.

\section{LA REPRÉSENTATION DE L'ESPACE QUI RESPECTE L'œIL. UNE RECHERCHE DE LA PROFONDEUR}

11 Avant cette "redécouverte» d'un espace poétique libre de toute contrainte, la Renaissance plaçant l'homme et donc son œil au centre du monde, parfois faisant du vecteur [point de fuite, œil du prince] le seul support possible à une construction de l'espace, a réduit la poïétique de l'espace à une obsession des proportions sous la domination d'un point de fuite. L'espace s'est fait mathématique, sa construction s'insère dans une grille géométrique, le simple point y possède des coordonnés. Le damier au sol et sa projection par rabattement sur un plan vertical dessinent un espace parallélépipédique filaire, une prison, une matrice qui détermine les segments, soit un début et une fin, une hauteur une largeur et évidemment une profondeur à toutes les choses reproduites. La perspective du Quattrocento est « une mise en ordre rationnelle du monde de la représentation $»^{22}$. Cette obsession de l'ordre, des proportions, d'une gestion harmonique du rapport entre les corps et les objets devait, pour Alberti, passer 
par une mise en scène véritable de la scène à reproduire, l'artiste a besoin de modèles ${ }^{23}$, il représente ce qu'il voit, d'un point de vue dans un cadre déterminé (la fenêtre Albertienne). «Par la perspective linéaire, on a donné un cadre rigide à la vision $»^{24}$. Pour André Bazin, «la perspective fut le péché originel de la peinture occidentale.» Elle a fermé le paradis de l'art médiéval ${ }^{25}$.

Cette domination de l'image figurative issue du Quattrocento répondant à une certaine logique constructive de l'espace, soit « un proche grand et un lointain petit » s'est imposée en Occident pendant plusieurs siècles. L'usage de la perspective linéaire, cette obligation de voir les choses dans les proportions liées à l'éloignement, cette acceptation de représenter ce qui se montre et non ce qui peut nous être caché, nous éloigne de la représentation symbolique des choses, d'une certaine poésie. "Notre vision et l'application des règles de la perspective sont le résultat d'un processus physique qui peut se reproduire, la chambre noire en est une reproduction technique, elle restitue des "simulacres se peignant sur le papier" ${ }^{26} .{ }^{27}$ Le choix du sujet à montrer ${ }^{28}$, à l'aide de cette "camera obscura ", est plus important que la façon dont on peut le reproduire, il faut juste montrer et non penser à interpréter.

13 Le cinéma doit se faire ciné-œil, celui de Dziga Vertov ${ }^{29}$ pour qui le cinéaste doit restituer, saisir la vie comme elle est ${ }^{30}$. La reproduction, l'interprétation nécessite un travail réfléchi, la mise en application de techniques de reproduction artistique, l'utilisation et la maitrise d'outils.

14 La boite noire, si elle peut s'apparenter à l'œil, n'a pas besoin de réfléchir ; d'ailleurs, l'œil d'un homme fraichement mort ou celui d'un bœuf peut reproduire en perspective les objets dressés devant cet œil technique ou physiologique ${ }^{31}$. Les prolongations technologiques de la vision humaine telles que la caméra, ${ }^{32}$ l'appareil photo ne deviennent intéressantes que maitrisées par certains artistes. Les artistes, à travers l'histoire de l'art, ont su se libérer de ce dictat, le simple cadrage est un acte constructeur et libératoire.

15 Il existe une filiation flagrante entre la peinture du Quattrocento, l'invention de la perspective, les installations des plasticiens travaillant l'espace, l'architecture et la vision comme Felice Varini et Georges Rousse. Les dispositifs spatiaux du décor de cinéma sont assujettis à cette perspective artificielle, en effet :

16 «Les images réalisées avec un appareil photographique ou une caméra pour être tirées sur papier ou projetées sur un écran sont soumises aux règles de la construction perspective des images planes. Très tôt, les réalisateurs et décorateurs de cinéma ont donc dû tenir compte des caractéristiques optiques de ces outils et des propriétés de la perspective à laquelle ils étaient confrontés $»^{33}$.

17 Ces propriétés de la perspective permettent de traiter l'espace dans la profondeur suivant un rythme d'espaces fractionnés, véritable construction anamorphique d'une réalité optique. Pour comprendre la structure des agencements fractionnés du décor de cinéma et de ces plasticiens, nous pouvons, comme le propose Merleau-Ponty ${ }^{34}$, prendre un regard latéral pour les appréhender et comprendre leur profondeur. Mais ce regard ne donnera pas accès au locus considéré par Albert le Grand, ce philosophe, frère dominicain du XIII , comme « un principe actif d'engendrement $»^{35}$, un lieu où la perspective architecturale et la perspective "psychique ${ }^{36}$ d'André Chastel due à un effet physiognomonique des gestes, s'entendent et composent pour raconter l'histoire. Une compréhension narrative de la scène fictive nécessite un ancrage à un endroit que 
je nomme "panoptique du sens" et, comme l'augure à travers le templum, nous attendons le passage des oiseaux.

Le trou percé dans la tavoletta de Filippo Brunelleschi ${ }^{37}$ montre un espace de structures et d'intervalles, un éloge de la profondeur. Ils sont tous deux déjà des agencements révélateurs de cet endroit "panoptique du sens ». La révélation d'une image implique une vision particulière, celle d'un spectateur. L'apparition, la disparition dans l'espace du baptistère feint ${ }^{38}$, le défilement des nuages, le chemin des nuées, sont des dispositifs de spectacle. La perspective linéaire s'est construite en fonction de cet «œilspectateur » et de sa projection. C'est une illusion d'espace montrée aux spectateurs, au regardeur ${ }^{39}$.

du théâtre de la Renaissance italienne est un dispositif où l'artiste fait acte d'autorité. L'artiste y impose sa vision comme le peintre face à son espace qui « sublime le plat en plan (avec ses avant-plans et ses arrière-plans) et y creuse une profondeur " ${ }^{40}$. Cette théorie du plan créateur de volume était déjà évoquée par Philippe de La Hire, en $1694^{41}$. Toute la construction spatiale de cette scène se fera autour de la place du prince et de son œil ${ }^{42}$, canalisé à travers le cadre de scène.

20 Cet agencement étalé dans la profondeur, ce diorama, ce pop-up est évidemment repris dans l'espace plastique du décor de cinéma et dans sa plastique sonore qui est parfois une construction spatiale fragmentée $\mathrm{du}$ son au service d'une imagination constructrice.

21 «Lors des différentes étapes de la production sonore, de la prise de son à la diffusion en passant par le montage, le traitement en postproduction et le mixage, la question de la représentation de l'espace se pose de façon toujours singulière et nécessite, outre la mise en œuvre de systèmes complexes, d'intégrer les mystérieuses mécaniques qui président chez l'auditeur ou l'audio-spectateur à la construction de la représentation de l'espace sonore. $»^{43}$ Mystérieuses Mécaniques d'une réception d'un flux sonore «dont nous sommes prisonniers $»^{44}$,

Une abondance d'informations qui nécessite un apprentissage de la scène sonore, un décryptage de la distance, de la profondeur.

C'est également dans la profondeur que Georges Méliès, en 1898, dans son film Visite sous-marine du Maine $e^{45}$, aura l'idée de placer entre la caméra et son décor, un aquarium dans lequel évoluent des poissons rouges, recréant ainsi les fonds marins. Ce geste important est sûrement fondateur de l'espace scénique du cinéma, il intègre la mise en œuvre de cette maquette de premier plan et de ce dispositif de matte-painting. Ce type d'agencement est impensable dans la configuration de la scène théâtrale. Il joue sur une règle de la perspective où un petit objet vu de près peut être aussi grand, voire plus grand qu'un grand objet vu de loin. Sa captation par l'œil-caméra bouleverse la règle de géolocalisation des éléments du dispositif, le loin peut-être proche de l'œil. Le dispositif cinématographique du Matte Painting, est l'exemple même de ces petits arrangements spatiaux. Ce trucage cinématographique doit pour fonctionner se fondre dans l'espace perspectif de la scène en captation. ${ }^{46}$ L'espace truqué se fera l'image d'un espace cohérent, rien ne viendra choquer cette dictature de l'œil. 


\section{De l'image à l'espace, et de l'espace à l'image, une ontogenèse à double sens} cadré par cet admoniteur architectonique qu'est le cadre de scène, est devenu imagetableau, une scéna-quadro ${ }^{47}$. Les dispositifs de Fernand Léger dans son film Ballet mécanique, les décors de Jacques Saulnier décorateur entre autres d'Alain Resnais, se sont plaqués contre le support souple de la pellicule, en un assemblage de photogrammes. Ils se sont fait image afin d'être projetés sur le plan de l'écran, «la transformation géométrique ainsi définie, opérant la contraction d'un espace géométrique à 3 dimensions (volume) sur un espace géométrique à 2 dimensions (le plan de l'image) $»^{48}$. Le miroir du dispositif de Filippo Brunelleschi qui réfléchit le baptistère peint de San Giovanni double l'image, cette "image - miroir» se fait «image-écran» elle prendra sa place dans l'espace. Le voile intersecteur de Léon Battista Alberti ${ }^{49}$ transforme l'espace de la scène vue en une captation plane, une image.

L'écran est nécessaire dans la mise en place de l'historia, il est ce point nodal situé à la frontière entre espace et image d'espace, «l'écran articule ces deux ordres de la représentation, le géométral qui régit l'espace et le symbolique qui construit le sens.» ${ }^{50}$. La perte d'une dimension, cette transformation de l'espace en image, c'est en cela que réside la magie, «la soustraction donne la force, c'est que la puissance naît de l'absence $\aleph^{51}$.

ette image révélée est donc issue de l'espace, mais il existe évidemment dans une pratique, entre autres d'atelier, de travail de recherche, le chemin inverse, où l'image peut se faire espace. Donc, l'espace devient image, et l'image peut se faire espace, et ainsi de suite, nous sommes face à une ontogenèse à double sens. L'espace pour le plasticien peut-être avant tout une image, cette image ${ }^{52}$ que l'on peut nommer « image de travail » peut prendre les formes d'un story-board, d'une esquisse, d'une image iconographique, d'un linéament comme embryon créatif. Chaque projet plastique doit s'accompagner de ces schèmes, l'ossature d'une esquisse, d'un croquis, images qui peuvent s'apparenter à une lointaine petite musique dans le silence. Ce corpus d'illustrations qui accompagne la conception de l'espace est à la fois le premier tracé au sol du Rex à l'aide de son bâton et la dernière brique du mur d'enceinte.

Pour l'architecte, l'image travaille peut-être un plan, une élévation, soit un autre type de fragmentation de l'espace, un "mode si particulier de figuration $»^{53}$. Face à cette image en deux dimensions, l'architecte doit retrouver l'espace qu'elle emprisonne dans sa surface, il doit faire un travail de décryptage, de visualisation. Pour le scénographe d'exposition, c'est l'assemblage fragmenté d'images (œuvres) qu'il nous donne à contempler et à découvrir qui se fera espace, nouvel espace de tensions où une œuvre répond à une autre. C'est à travers un collage, un montage spatial des œuvres, qu'il devient un designer de vie ${ }^{54}$. Comme le collectionneur ${ }^{55}$, il accapare les images des autres pour en faire une image spectacle, l'image partielle d'une œuvre, un moment de la vie de l'artiste, et à "l'instar du lecteur, le spectateur part à l'assaut de la fragmentation et réécrit l'exposition dans l'espace de son imaginaire $»^{56}$.

Le développement de certains programmes informatiques permet à partir d'une image photographique et d'une vision par ordinateur de reconstruire un espace 
tridimensionnel, là où face à une image, j'imaginais l'espace qu'elle représente, des calculs informatiques me le restituent. Il serait donc facile de modéliser n'importe quel « espace plat » d'une image, d'y prendre possession de l'espace qu'elle emprisonne ${ }^{57}$. Il serait donc possible de déambuler dans les structures spatiales de Maurits Cornelis Escher, et de s'y perdre,

Mais ce n'est pas si simple que cela et il faut analyser l'œuvre avec précision si l'on veut effectivement l'adapter par ordinateur, car Escher est très habile mélangeant perspective classique et axonométrie, illusions d'optique et études réalistes : il ne s'agit pas chez lui d'illustrer des illusions d'optique, mais de les transformer en une réflexion sur l'espace et le temps. ${ }^{58}$

Passez donc de l'image à l'espace, insufflez de l'air dans cette «double réalité des images $"{ }^{59}$. Insuffler exprime le fait de donner la vie. L'image peut alors se faire lieu, locus au sens archéologique ou théâtral, soit un réceptacle à actions ou à traces de vie. Mais n'existe-t-il pas des images, des dessins, des reproductions d'espaces métaphysiques, une abstraction d'espace, qui se refusent à donner lieu ? La modélisation d'une anamorphose, d'une illusion accepte une lecture de ce point "panoptique du sens », mais ne permet pas un déplacement, une visite. Le lieu y reste narratif, lisible par un spectateur immobile.

\section{NOTES}

1. DE LA SOUCHÈRE Marie-Christine, Quand la vue change de sens. Revue La Recherche $\mathrm{N}^{\circ} 443$ 06/2010. "L'unité de fonctionnement des cinq sens plaidait aussi en faveur d'un œil récepteur. L'ouie, le toucher, l'odorat et le goût s'expliquaient par une réaction à des stimuli externes. Le son partait à la rencontre de l'oreille, les parfums allaient au-devant du nez.Il n'y avait aucune raison que la vue échappât à la règle. »

2. SIMON Gérard, Archéologie de la vision, L'optique, le corps, la peinture, Paris : Éditions du Seuil, 2003 , p. 89.

3. FRANCASTEL Pierre, L'image la vision et l'imagination : l'objet filmique et l'objet plastique, Paris : Éd Denoël- Gonthier, 1983, p.161. « On ne peut pas dire que le jour où les artistes ont trouvé la perspective linéaire, l'art a été changé et que ce fut un progrès absolu. C'est absolument faux».

4. FRANCASTEL Pierre, L'image la vision et l'imagination : l'objet filmique et l'objet plastique, Paris : Éditions Denoël-Gonthier, 1983, p. 210, p. 49.

5. HEGEL Georg Friedrich Wilhelm, Esthétique, Tome premier, 1835, Traduction française de Ch. Bénard, Un document produit en version numérique par Daniel Banda, Dans le cadre de la collection : "Les classiques des sciences sociales" dirigée et fondée par Jean-Marie Tremblay, p. 28. « Or à quoi bon reproduire ce que la nature déjà offre à nos regards ? Ce travail puéril, indigne de l'esprit auquel il s'adresse, indigne de l'homme qui le produit, n'aboutirait qu'à lui révéler son impuissance et la vanité de ses efforts ; car la copie restera toujours au-dessous de l'original. D'ailleurs, plus l'imitation est exacte, moins le plaisir est vif. Ce qui nous plaît, c'est non d'imiter, mais de créer. La plus petite invention surpasse tous les chefs-d'œuvre d'imitation. »

6. PANOFSKY Erwin, Idea, Paris : Editions Gallimard, 1989, pp. 58-60, «...pour le Moyen Âge, l'œuvre d'art ne résulte pas d'une explication entre l'homme et la nature... ...mais de la projection dans la matière d'une image intérieure", p.60. "C'était donc, pour la pensée 
médiévale, un fait solidement établi que l'artiste créait des formes en s'inspirant, sinon d'une idée au sens proprement métaphysique, du moins d'une représentation de la forme, intérieure à l'artiste lui-même et préexistant à l'œuvre... », p. 58.

7. LUCRÈCE, De la nature, livre IV, Paris : Edition Les Belles Lettres, 1984, 1985, pp. 170-171.

8. France Culture, La grande traversée, L'étonnant Monsieur Resnais par Antoine Guillot, juillet, août 2014. «...mais j'ai l'impression quand je tourne, pour que j'aie du vrai plaisir, il faut que je me sente dans le camp Méliès »

9. Interview d'Alain Resnais, Images pour un film La vie est un roman, in, THEVENET Jean-Marc, Images pour un film, les décors d'Enki Bilal pour la vie est un roman d'Alain Resnais, Paris : Éditions Dargaud Editeur, 1983, p. 16.

10. Par le Portillon d' Albrecht Dürer, ou le viseur de la chambre photographique.

11. BARTHES Roland, Euvres complètes, Tome V, textes 1978, Arcimboldo ou Rhétoriqueur et magicien. Paris : Éditions du Seuil, 1978, pp. 496-497.

12. FERGOMBÉ Amos, Scénographie et mise en abyme de l'intime, in Espace, Perspective et Fragmentation. «...2002, année de sa première mise en scène de l'Affaire Elseneur ou le meurtre de Gonzague du metteur en scène et acteur bulgare Nedjalko Jordanov. »

13. FERGOMBÉ Amos, Scénographie et mise en abyme de l'intime, in Espace, Perspective et Fragmentation.

14. BARRÈS Patrick, Boîte noire, une "scène d'invention totale ", in Espace, Perspective et Fragmentation. 15. BARRÈS Patrick, Boîte noire, une "scène d'invention totale ", in Espace, Perspective et Fragmentation. "...des figures d'ombre prennent forme et se déforment sans cesse, suivant les mouvements des pièces sur la scène, au contact des foyers de lumière. »

16. LEGER Fernand, Fonctions de la peinture, Paris : Gallimard, 1997, p. 28 et p. 119

17. (Définition de Panofsky)

18. NICOARA Marie-Garé, Désarticuler la figure humaine : boites d'espaces et boites à images chez Teatrocinema (Histoire d'amour (2013), Sans sang (2007) et L'homme qui donnait à boire aux papillons (2010)), in Espace, Perspective et Fragmentation.

19. BERQUE Augustin, Paysage à la chinoise, paysage à l'européenne, in MOTTET Jean (sous la direction), Les paysages du cinéma, Seyssel : Éditions Champ Vallon, 1999, p. 62.

20. BERQUE Augustin, Paysage à la chinoise, paysage à l'européenne, in MOTTET Jean (sous la direction), Les paysages du cinéma, Seyssel : Éditions Champ Vallon, 1999, p. 64.

21. Il s'agit de pensées rapportées par le peintre et critique Émile Bernard dans son article «Paul Cézanne ", publié dans la revue L'Occident, $\mathrm{n}^{\circ} 32$, juillet 1904.

22. SEGUIN Louis, L'espace du cinéma (Hors-champ, Hors-d'œuvre, Hors- jeu), Toulouse : Éditions Ombres, 1999, p. 40.

23. PANOFSKY Erwin, Idea, Paris: Editions Gallimard, 1989, p. 63. Avec la Renaissance, « Quelque chose d'extraordinairement neuf apparaît: le peintre reçoit le conseil de se placer face à un modèle ».

24. FRANCASTEL Pierre, L'image la vision et l'imagination : l'objet filmique et l'objet plastique, Paris : Éditions Denoël- Gonthier, 1983, p. 210.

25. Ibid.

26. CHONÉ Paulette, Le P. Levrechon et l'image cinétique "représenter en une chambre close tout ce qui se passe dehors, in, WUNENBURGER Jean-Jacques, (Sous la direction), La Renaissance ou l'invention d'un espace, Dijon : Éditions Universitaires de Dijon, collection figures libres, 2000, p. 261.

27. BLED Grégory, De l'image à l'espace, le regard du décorateur de cinéma, in Espace, Perspective et Fragmentation.

28. CHONÉ Paulette, Le P. Levrechon et l'image cinétique "représenter en une chambre close tout ce qui se passe dehors, in, WUNENBURGER Jean-Jacques, (Sous la direction), La Renaissance ou l'invention d'un espace, Dijon : Éditions Universitaires de Dijon, collection figures libres, 2000, p. 261.. « Il faut 
d'abord, recommande notre ami Levrechon, choisir « une chambre qui regarde sur quelque place, ou rue fréquentée, sur quelque beau bâtiment, ou parterre florissant, pour avoir plus de plaisir ». 29. VERTOV réalisateur soviétique 1896-1954, il est resté dans l'histoire du cinéma comme l'initiateur de l'école des documentaristes russes.

30. FERNANDEZ Dominique, Eisenstein, Paris: Éditions Grasset et Fasquelle, 1975, p. 83.

31. HAVELANGE Carl, De l'œil et du monde, une histoire du regard au seuil de la modernité, Paris : Librairie Arthème Fayard, 1998, p. 331. Carl Havelange cite des passages du livre Dioptrique de René Descartes : Descartes, dans la Dioptrique, première illustration du discours de la méthode, écrite d'ailleurs avant le Discours lui-même, reprend bien entendu à son compte le modèle de l'image rétinienne et sans rien y apporter de neuf qui soit vraiment fondamental. A propos des « images qui se forment sur le fonds de l'œil », Descartes évoque ceux-là qui s'en sont « désia tresingenieusement expliqué, par la comparaison de celles qui paroissent dans une chambre, lors que l'ayant toute fermée, réservé un seul trou,\& ayant mis au-devant de ce trou un verre en forme de lentille, on estend derriere, à certaine distance, un linge blanc, sur qui la lumiere, qui vient des objets du dehors, forme ces images. Car ils disent que cette chambre représente l'œil ; ce trou, la prunelle; ce verre, l'humeur cristalline, ou plustost toutes celles des parties de l'œil qui causent quelque refraction; \& ce linge, la peau intérieure, qui est composée des extremités du nerf optique » (page 35) Mieux encore, en s'aidant de l'œil d'un « homme fraichement mort », ou, « au defaut, celuy d'un bœuf ", on pourra, après une adéquate préparation anatomique, faire apparaître « non peust-estre sans admiration, \& plaisir, une peinture, qui représentera fort naïvement en perspective tous les objets » (page 37) .

32. BONITZER Pascal Décadrages. Peintures et cinéma, Paris : Cahiers du cinéma Éd de L'étoile, 1995. p. 14. "La caméra produirait un code perspectif hérité du Quattrocento, il ne s'ensuit pas nécessairement qu'elle n'entretient aucun rapport avec le réel, puisque si la perspective du Quattrocento peut être dite scientifique (par Pleynet lui-même), c'est parce qu'elle mathématise les conditions de la perception oculaire, donc donne un statut partiellement objectif à la représentation » Pascal Bonitzer évoque là le problème qui on l'a vu a tourmenté la Renaissance entre une vision monoculaire et binoculaire qui serait considérée comme une vision réelle. Mais pour Mitry, la caméra « rapporte objectivement le réel visé. »

33. ANTONUCCI Jean-Luc, Perspective et constructions, in Espace, Perspective et Fragmentation.

34. MERLEAU-PONTY Maurice, Phénoménologie de la perception, Paris : Éditions Gallimard, 1945, pp. 295-296. «Ce que j'appelle profondeur est en réalité une juxtaposition de points comparables à la largeur. Simplement, je suis mal placé pour la voir. Je la verrais si j'étais à la place d'un spectateur latéral, qui peut embrasser du regard la série des objets disposés devant moi, tandis que pour moi ils se cachent l'un l'autre ».

35. DIDI-HUBERMAN Georges, Fra Angelico, Dissemblance et figuration, Paris : Éditions Flammarion, 1995, p. 34.

36. CHASTEL André, Le geste dans l'art, Paris : Éditions Liana Levi, 200, pp. 16-17.

37. ANTONUCCI Jean-Luc, Perspective et constructions in Espace, Perspective et Fragmentation. « Pour la première expérience, Filippo Brunelleschi avait peint sur une tavoletta (panneau de bois) d'une trentaine de centimètres de côté, le Baptistère qui fait face au portail de la cathédrale Sainte Marie des Fleurs. Le bâtiment était peint tel qu'on pourrait le voir si on se plaçait dans l'encadrement du portail de la cathédrale. Le panneau était percé d'un trou à travers lequel on regardait l'image peinte du baptistère réfléchie dans un miroir que l'on tenait à bout de bras. »

38. PERELMAN Marc, Construction du corps, Fabrique de l'architecture, Figures, Histoire, Spectacle, Paris : Les Editions de la Passion, 1994, p. 65. «La mise en place de l'expérience oblige à se cacher le visage, à se « voiler la face »... ...Cette action met en avant l'œil [...] L'œil qui ressortit à une puissance bien plus importante, voire vertigineuse, que le visage lui-même ; ce dernier subissant une sorte d'éclipse, d'oppression, ce que l'on peut appeler une aliénation dans l'ordre du voir. Devant le visage, il y a la vision. Le visage ne transforme rien, la vision, elle, se transforme en 
prise, en une possession. D'où sa force devenue par la suite invincible. D'où peut-être, par anticipation, ce retrait du visage par rapport à la vision. Celui-là est aliéné, celle-ci est en plein devenir»

39. Pour Marcel Duchamp c'est le regardeur qui fait l'œuvre, le tableau.

40. GUÉRIN Michel, L'espace plastique, Bruxelles : Éditions La part de l'œil, 2008, p. 94.

41. En 1694 Philippe de La Hire, théoricien de l'architecture, nous précise que, pour rendre l'impression de volume d'un élément architectural, il suffit de représenter sur des tableaux légèrement espacés chaque partie de cet élément ne se trouvant pas sur le même plan.

42. MARCIAK Dorothée, La place du prince, Perspective et pouvoir dans le théâtre de cour des Médicis, Florence (1539-1600), Paris : Éditions Champion, 2005, p. 194. «Placé en position de démiurge (il est le double métaphorique du décorateur concepteur de scènes), le prince est à l'endroit d'où toutes choses se donnent à voir dans leur perfection naturelle "

43. BEZNOSIUK Alexandre, in Espace, Perspective et Fragmentation.

44. BEZNOSIUK Alexandre, in Espace, Perspective et Fragmentation.

45. MÉLIÈS Georges, Visite sous-marine du Maine, (Divers at Work on the Wreck of the "Maine»), $1 \mathrm{~mm}$, Production: Star Film, 1898.

46. HAMUS-VALLEE Réjane, Le Matte Painting : Du trompe-l'œil au trompe la perspective, in Espace, Perspective et Fragmentation. "...l'obligation d'avoir une perspective unique contraint les matte painters à reproduire scrupuleusement les lignes de fuite déjà présentes dans l'image cinématographique, référence absolue de leur travail pictural ».

47. SURGERS Anne, D'une «Scène-Tableau » à une « Scène Théâtre " quelques pistes pour une lecture des adaptations du modèle à l'Italienne en France au XVII ${ }^{e}$ siècle, in, HAQUETTE Jean-Louis, HÉNIN Emmanuelle, Etudes réunies, et travaux du groupe CLAM de l'Université de Jussieu Paris 7. La scène comme tableau, Poitiers : La licorne, UFR de Langues et Littératures, 2004, p. 7. Pour Anne Surgers, « l'apparente intégration du corps de l'acteur à la perspective a permis que naisse la notion de la scena-quadro. Il est d'usage, en français de traduire scena-quadro par scène-tableau ». Anne Surgers précise que cette traduction est erronée. Scena signifie décor donc nous sommes face à un décor-tableau.

48. ANTONUCCI Jean-Luc, Perspective et constructions in Espace, Perspective et Fragmentation.

49. ALBERTI Léon Batista, De la Peinture, De Pictura (1435) Traduction Jean Louis Schefer, préface Jean Louis Schefer, introduction Sylvie Deswarte-Rosa, Collection La Littérature Artistique, Paris : Éditions Macula Dédale, 1992, L2, \$31, p. 147 «Il faut ainsi s'appliquer à ce tracé des contours et pour l'obtenir parfaitement, je crois qu'on ne peut rien trouver de plus pratique que ce voile que j'ai l'habitude avec mes amis d'appeler « intersecteur »...»

50. LOJKINE Stéphane, (sous la direction de), L'écran de la représentation, Paris: Éditions L'Harmattan, Champs visuels, 2001, p. 13.

51. BAUDRILLARD Jean, Illusion, désillusion esthétiques, Paris : Éditions Sens \& Tonka, 1997, pp. 61-62 « Pornographie de l'image à trois ou quatre dimensions, de la musique à trois ou quatre ou vingt-quatre pistes - c'est toujours en ajoutant au réel, en ajoutant le réel au réel dans l'objectif d'une illusion parfaite (celle de la ressemblance, celle du stéréotype réaliste parfait) qu'on tue justement l'illusion en profondeur. Le porno, en ajoutant une dimension à l'image du sexe, en ôte une à la dimension du désir et disqualifie toute illusion séductrice(...), p. 61 (...) ce que nous avons désappris de la modernité : c'est que la soustraction donne la force, c'est que la puissance naît de l'absence. Nous n'avons de cesse d'accumuler, d'additionner, de surenchérir. Et que nous ne soyons plus capables d'affronter la maîtrise symbolique de l'absence fait que nous sommes aujourd'hui plongés dans l'illusion inverse, celle désenchantée et matérielle de la profusion, l'illusion moderne des écrans et des images, p. 62.

52. FRANCASTEL Pierre, L'image la vision et l'imagination : l'objet filmique et l'objet plastique, Paris : Éd Denoël- Gonthier ,1983. p. 22. Il faut penser l'image, tel que nous le conseille Pierre Francastel, comme des structures signifiantes qui peuvent nous fournir un grand nombre d'informations, 
nous provoquer un grand nombre de sensations. Elles nous parlent « suivant leurs principes propres »

«En montrant une chose on fournit des informations aussi bien abstraites que sensibles d'une précision au moins égale, mais sur d'autres plans, aux informations du discours. La mise en combinaison artificielle d'éléments matériels servant de support à des perceptions visuelles ou sonores n'est pas moins signifiante que l'ordre combinatoire des mots ».

53. ESTEVEZ Daniel, Représentation dissensuelle en architecture, in, Espace, Perspective et Fragmentation. «Or ce mode si particulier de figuration défini par le système géométral ne paraît pourtant pas approprié à la recherche de cohérence et de coordination que constitue la conception ou même l'analyse du projet. Le dessin géométral, en effet, est caractérisé non seulement par sa bidimensionnalité mais aussi par sa fragmentation (décomposition en plan/ coupe/élévation). Deux propriétés contraignantes qui vont à l'encontre de l'objectif que se fixe chaque architecte lors de l'élaboration de son projet : en contrôler et en maîtriser l'évolution dans sa globalité et sa complexité (volumétrique, compositionnelle etc.). Comment interpréter ce «paradoxe du géométral»?

54. Pour Michel Antaki « Toute construction de vie est un design, ce qui meuble la construction de la vie c'est la mémoire, et ce qui est dans la mémoire c'est les objets »

55. Le scénographe est le collectionneur, qui devient dès lors l'artiste privilégié de par sa propre collection. "C'est un super artiste qui utilise nos œuvres pour composer sa propre toile " déclarait Daniel Buren à propos de son ami et commissaire d'exposition Harald Szeemann (ce dernier s'est illustré comme commissaire indépendant et scénographe d'exposition dès la fin des années 50). De l'ensemble des œuvres qu'il exposait, il recréait une nouvelle œuvre d'art, Michel Antaki, MAMAC Liège, 2012.

56. JOURDAIN Frédéric, La scénographie à l'épreuve de la fragmentation des espaces d'exposition. Alternative à l'immersion du spectateur et à la dramatisation des ouvres, in Espace, Perspective et Fragmentation.

57. AUMONT Jacques, L'œil interminable, Paris : Nouvelles Éditions Séguier, 1995, ppp. 138-139-141. "Voir l'espace, ce serait donc nécessairement interpréter, au prix d'une construction déjà complexe, un certain nombre d'informations visuelles. Au premier chef celles relatives à ce qu'on appelle couramment " profondeur » ou " troisième dimension » de l'image, manifestant d'ailleurs ainsi suffisamment que l'espace est d'abord rapporté au corps, à sa hauteur- première dimension, à sa largeur- deuxième dimension, la profondeur étant la dimension de l'avancée virtuelle du corps entier .

Jacques Aumont revient dans ce livre sur la véritable contradiction perceptive que nourrit le tableau :

L'espace " tridimensionnel » représenté y étant mis sur un pied d'égalité avec l'espace, réellement bidimensionnel, de la toile. C'est exactement cette contradiction, et presque cette compétition, entre deux types de perception à priori incompatibles, que désigne la notion proposée par Maurice Pirenne, de "double réalité " perceptive des images. Double réalité, puisque l'œil perçoit en même temps l'espace plat de la surface de la toile et la vue partielle sur un fragment d'espace « en profondeur » produit en autres par l'emploi de la perspective. Double réalité puisque l'un et l'autre de ces espaces sont réellement perçus, et, jusqu'à un certain point, perçus comme réel »

58. METHEL Gilles, Un art magique?, in, Espace, Perspective et Fragmentation.

59. AUMONT Jacques, L'image, Paris : Éditions Nathan, cinéma, 1999, p. 42. « Autrement dit, nous percevons simultanément cette image comme un fragment de surface plane et comme un fragment d'espace tridimensionnel : c'est ce phénomène psychologique fondamental que l'on appelle double réalité perspective des images ou par abréviation, double réalité des images. 
Les deux « réalités » de l'image ne sont pas du tout de même nature dans l'absolu, puisque l'image comme portion de surface plane est un objet qui peut se toucher, se déplacer, et se voir, alors que l'image comme portion du monde en trois dimensions existe uniquement par la vue »

\section{AUTEUR}

\section{GRÉGORY BLED}

Décorateur, Docteur en Cinéma, Professeur Associé à l'ESAV, chercheur au LARA-SEPPIA, Université de Toulouse Jean Jaurès, France. 